From the above and from (8), we obtain

$$
\left\{\frac{1-\alpha}{r}\right\}=\left\{\frac{\alpha}{r}\right\}
$$

and from (7) it follows that

$$
r^{p-1} \equiv 1
$$

As before, a similar proof obtains when $y$ is divisible by $p$.

Ottawa, Canada

\title{
ON THE SOLUTION OF THE EULER EQUATIONS FOR THEIR HIGHEST DERIVATIVES*
}

\author{
BY H. V. CRAIG
}

1. Introduction. J. H. Taylor $\dagger$ has given two elegant methods of solving for their highest derivatives the Euler equations associated with the integral $\int F(x, \dot{x}) d t$. In this paper these two methods are modified so as to apply to the more general case in which the Euler equations contain derivatives of order higher than the second.

2. Notation. Throughout this paper we shall employ vector notation and shall use dots and enclosed superscripts to indicate differentiation with respect to the parameter. Thus $x, \dot{x}, x^{(m)}$ will stand for the sets

$$
x^{1}, x^{2}, \ldots, x^{n} ; \frac{d x^{\prime}}{d t}, \frac{d x^{2}}{d t}, \ldots, \frac{d x^{n}}{d t} ; \frac{d^{m} x^{1}}{d t^{m}}, \frac{d^{m} x^{2}}{d t^{m}}, \ldots, \frac{d^{m} x^{n}}{d t^{m}}
$$

respectively. Partial derivatives will be denoted by means of subscripts, thus

* Presented to the Society, September 7, 1928. This paper is a part of a thesis written at the University of Wisconsin under the direction of Professor J. H. Taylor.

$\dagger \mathrm{J} . \mathrm{H}$. Taylor, The reduction of Euler's equations to a canonical form, this Bulletin, vol. 31 (1925) p. 257. 


$$
\begin{aligned}
& \frac{\partial F\left(x, \dot{x}, \cdots, x^{(m)}\right)}{\partial x^{i}}=F_{i^{0}} ; \quad \frac{\partial F\left(x, \dot{x}, \cdots, x^{(m)}\right)}{\partial \dot{x}^{i}}=F_{i^{1}} ; \\
& \frac{\partial F\left(x, \dot{x}, \cdots, x^{(m)}\right)}{\partial \ddot{x}^{i}}=F_{i^{2}} ; \cdots .
\end{aligned}
$$

However, if the differentiation is with respect to the highest derivatives present we shall further abbreviate by omitting the $m$, that is,

$$
\frac{\partial F\left(x, \dot{x}, \cdots, x^{(m)}\right)}{\partial x^{i(m)}}=F_{i} .
$$

Summations are to be understood when repeated indices occur.

3. The Calculus of Variations Problem and the Euler Equations. We consider a function $F\left(x, \dot{x}, \cdots, x^{(m)}\right), m>1$, with properties to be specified and seek among all curves of class $2 m$ lying in a certain region of an $n$-space and satisfying certain boundary conditions, the one which gives the integral

$$
I=\int_{t_{1}}^{t_{2}} F\left(x, \dot{x}, \cdots, x^{(m)}\right) d t
$$

its minimum value.

As a first hypothesis on $F$ we suppose, as just implied, that the solution of this problem exists uniquely. The additional hypotheses are: (a), that $F$ is of class $m+1$; (b), that the classical $\bar{F}_{1}$ function* associated with $F$ does not vanish along the solution; and (c), that $I$ is independent of the choice of the parameter.

Zermelo has shown $\dagger$ that this invariance of $I$ implies the following identities in $x, \dot{x}, \cdots, x^{(m)}$ :

$$
\begin{aligned}
\dot{x}^{i} F_{i} & \equiv 0, \quad i \text { ranges from } 1 \text { to } n, \\
x^{i(\alpha)} E_{\alpha i} & \equiv F, \quad \alpha \text { ranges from } 1 \text { to } m,
\end{aligned}
$$

* We have used $\bar{F}_{1}$ to avoid confusion with $F_{1}=\partial F / \partial x^{1(m)}$. For a discussion of the $\bar{F}_{1}$ function, see Oscar Bolza, Vorlesungen über Variationsrechnung, Leipzig, Teubner, 1909, p. 13. Oscar Bolza, Lehrbuch der Variationsrechnung, p. 196.

† See Adolph Kneser, Lehrbuch der Variationsrechnung, Leipzig, Teubner, 1925, p. 217. 
where $E_{q i}$ is defined by the equation

$$
E_{q i} \equiv(-1)^{\beta} F^{\beta}{ }^{q} q+\beta, \quad(\beta \text { ranges from } 0 \text { to } m-q) .
$$

We observe from this definition that $E_{0 i}=0, i=1,2, \cdots, n$, are the Euler equations associated with $F$. These equations are not independent, but satisfy the relation

$$
\dot{x}^{i} E_{0 i}=0 .
$$

This may be established as follows. Differentiating (2) we obtain

$$
\dot{F}=x^{i(\alpha)} \dot{E}_{\alpha i}+x^{i(\alpha+1)} E_{\alpha i} .
$$

If in this we replace the second term by its value as given by the formula

$$
E_{q i}=F_{i q}-E_{q+1 i}
$$

the relation becomes

$$
\dot{F}=x^{i(\alpha)}\left[F_{i}^{\alpha-1}-E_{\alpha-1 i}\right]+x^{i(\alpha+1)} E_{\alpha i} .
$$

Since $E_{m i}=F_{i}$, this may finally be written

$$
\dot{F}=x^{i(\alpha)} F_{i}{ }^{\alpha-1}+x^{i(m+1)} F_{i}-\dot{x}^{i} E_{0 i}+x^{i(\alpha)} E_{\alpha-1 i}-x^{i(\alpha)} E_{\alpha-1 i} ;
$$

hence $\dot{x}^{i} E_{0 i}=0$.

Since (1) evidently leads to

$$
\dot{x}^{i} F_{i j}=0, \quad(j=1,2, \cdots, n),
$$

it follows that the determinant $\left|F_{i j}\right|$ (and this is the determinant of the coefficients of $x^{(2 m)}$ in the Euler equations) vanishes. Accordingly, the problem of solving the Euler equations for their highest derivatives requires special consideration. We shall make it a part of our hypotheses on $F$ that the rank of this determinant be $n-1$.

For use in determining the rank of certain determinants which will appear presently, we insert here a few miscellaneous observations. As a consequence of equation (4) and the rank of the determinant $\left|F_{i j}\right|$, the cofactors $F^{i j}$ of the latter satisfy the following relations:

$$
\frac{\dot{x}^{1}}{F^{i 1}}=\frac{\dot{x}^{2}}{F^{i 2}}=\cdots=\frac{\dot{x}^{n}}{F^{i n}} .
$$


If we note that the quantities $F^{i i}$ are symmetric in their indices, these equalities are seen to be expressible in the form

$$
\frac{\dot{x}^{i} \dot{x}^{j}}{F^{i j}}=\frac{\dot{x}^{k} \dot{x}^{l}}{F^{k l}}
$$

where $i, j, k, l$ may each be any number of the set $1,2, \cdots, n$ and no summation is to be understood. The reciprocal of the common value of the members of $(5)$ is the $\bar{F}_{1}$ function of our problem.

4. The First Method of Solution.* Let $H(x, \dot{x})$ be any function of class $2 m-1$, homogeneous of degree plus one in $\dot{x}$, and nonvanishing along the solution of our problem. With these restrictions we may so select the parameter that $H$ will maintain the value unity along the solution. Differentiating the equation $H=1,2 m-1$ times with respect to $t$, yields the relation

$$
x^{i(2 m)} H_{i}+r=0 \text {. }
$$

Here we have written explicitly only the terms in $x^{(2 m)}$ and have represented by $r$ the remaining terms. This equation we adjoin to the system

$$
x^{i(2 m)} F_{i j}+w H_{j}+R_{j}=0,
$$

$R_{j}$ being so chosen that these relations reduce to the Euler equations for $w=0$. The system $(6,7)$ is linear in the variables $x^{(2 m)}$ and $w$, has a non-vanishing determinant, and determines the same set of values for $x^{(2 m)}$ as the Euler equations. To prove this last statement we multiply the equations of the set (7) by $\dot{x}^{j}$ and sum. Because of (3) and the conditions imposed on $H$ (the homogeneity of $H$ implies $\dot{x}^{i} H_{i}=H$ ) the result is $w=0$. The determinant of the system $(6,7)$ is equal to

$$
\pm H_{i} H_{j} F^{i j}= \pm \bar{F}_{1} \dot{x}^{i} \dot{x}^{j} H_{i} H_{j}= \pm \bar{F}_{1} \neq 0 \text {. }
$$

5. The Second Method of Solution. Let us replace the function $F\left(x, \dot{x}, \cdots, x^{(m)}\right)$ of our calculus of variations problem with a new function $f\left(x, \dot{x}, \cdots, x^{(m)}\right)$, which we define as follows:

* Taylor points out the incidence of this method in an article entitled The properties of curves in space which minimize a definite integral, by Mason and Bliss, Transactions of this Society, vol. 9 (1908), p. 443. 
$f\left(x, \dot{x}, \cdots, x^{(m)}\right) \equiv F\left(x, \dot{x}, \cdots, x^{(m)}\right)+\frac{1}{2}\left\{\frac{d^{m-1} H(x, \dot{x})}{d t^{m-1}}\right\}^{2}$.

We restrict the function $H$ as in the preceding section and select the parameter as before so that $H$ maintains the value unity along the unique solution $C$.

For such a parameter it is evident that

$$
\int_{C} f\left(x, \dot{x}, \cdots, x^{(m)}\right) d t=\int_{C} F\left(x, \dot{x}, \cdots, x^{(m)}\right) d t,
$$

and

$$
\int_{\bar{C}} f d t>\int_{C} F d t,
$$

if $\bar{C}$ is any other admissible curve. Therefore the curve $C$ is also an extremal of the integral $\int f d t$. (The problem associated with $f$ is not a Weierstrass problem since $t$ has a special meaning.) Furthermore the determinant $\left|f_{i j}\right|\left(f_{i j}=F_{i j}+H_{i} H_{j}\right)$ is different from zero since

$$
\left|f_{i j}\right|=H_{i} H_{j} F^{i j}=\bar{F}_{1} \dot{x}^{i} \dot{x}^{j} H_{i} H_{j} \neq 0 .^{*}
$$

Hence the values of the quantities $x^{(2 m)}$ along any extremal may be obtained by solving the Euler equations associated with $f$ by Cramer's rule.

It can be shown that the left members of the Euler equations in the unsolved form are the components of a covariant vector. The method of solution outlined above gives us a simple contravariant description of this vector.

The University of Texas

* See J. H. Taylor, loc. cit. p. 261, for the development of a similar deter. minant. 